\title{
The Evolution of the Oxygen Abundance in the Galaxy
}

\author{
Francesca Matteucci \\ Dipartimento di Astronomia, Universita' di Trieste, Via G.B. Tiepolo \\ 11, 34124 Trieste, Italy
}

Cristina Chiappini

Osservatorio Astronomico di Trieste, Via G.B. Tiepolo 11, 34124, Trieste, Italy

\begin{abstract}
We discuss the evolution of the $[\mathrm{O} / \mathrm{Fe}]$ as a function of $[\mathrm{Fe} / \mathrm{H}]$ in the Galaxy. In particular, we discuss the influence of different stellar yields as well as different SN (II, I) rates on the $[\mathrm{O} / \mathrm{Fe}]$ ratio. Possible explanations for the steep rise of the $[\mathrm{O} / \mathrm{Fe}]$ ratio at low metallicities, as indicated by recent observations, are critically discussed.
\end{abstract}

\section{Introduction}

In the past years a great deal of observational work has been devoted to measure the relative abundances of $\alpha$-elements to iron in stars in the local disk. A large compilation of data relative to $\mathrm{C}, \mathrm{N}, \alpha$-elements and $\mathrm{Fe}$ can be found in Chiappini et al. (1999). The data reveal that $\alpha$-elements $(\mathrm{O}, \mathrm{Mg}, \mathrm{Si}, \mathrm{Ca}, \mathrm{S})$ show an overabundance relative to $\mathrm{Fe}([\alpha / \mathrm{Fe}]>0)$ in metal poor stars $([\mathrm{Fe} / \mathrm{H}]$ $<-1.0$ ), whereas these ratios decrease for disk stars until they reach the solar value. This trend of the $\alpha$-elements relative to $\mathrm{Fe}$ is generally interpreted as due to the time-delay in the iron production which originates from long living white dwarfs in binary systems eventually exploding as type Ia SNe. As a consequence of this, we expect the $[\alpha / \mathrm{Fe}]$ ratios to be high and reflect the $\alpha / \mathrm{Fe}$ production ratio from massive stars at low metallicities. The available data for $\mathrm{Mg}, \mathrm{Si}, \mathrm{Ca}$ and $\mathrm{S}$ show that for $[\mathrm{Fe} / \mathrm{H}]<-1.0$ the $[\alpha / \mathrm{Fe}]$ ratio is almost constant (plateau), whereas for oxygen there seems to be a slight increase of the $[\mathrm{O} / \mathrm{Fe}]$ ratio with decreasing metallicity. This is visible in a recent compilation of data from Gratton et al. $(1996,2000)$, where $O$ is measured from the [OI] $\lambda \lambda$ 6300 line (see also Sneden this conference). On the other hand, recent papers (Israelian et al. 1998; Boesgaard et al. 1999), where the oxygen abundance derives from the UV OH lines, the only ones observed at low metallicities, show a steep increase of the $[\mathrm{O} / \mathrm{Fe}]$ ratio with decreasing $[\mathrm{Fe} / \mathrm{H}]$. This trend, if real, is difficult to reconcile with our knowledge about stellar nucleosynthesis since $\mathrm{O}$ should behave similarly to the other $\alpha$-elements.

In this paper we discuss the nucleosynthetic origin of oxygen and predict the $[\mathrm{O} / \mathrm{Fe}]$ versus $[\mathrm{Fe} / \mathrm{H}]$ behaviour by means of a detailed model of Galactic chemical evolution. Then we discuss the possible interpretations of the peculiar behaviour of oxygen, if real. 


\section{Where Do Elements Form?}

The oxygen and the other $\alpha$-elements $(\mathrm{Ne}, \mathrm{Mg}, \mathrm{Si}, \mathrm{S}, \mathrm{Ca}$ and $\mathrm{Ti})$ are mainly produced during the hydrostatic burning phases in massive stars $\left(M \geq 10 M_{\odot}\right)$. Their yields depend crucially on the pre-supernova model (convection criterion, mixing processes, mass loss and nuclear reaction rates). The oxygen and the other $\alpha$-elements are normally restored into the interstellar medium (ISM) via SN explosion (type II SNe). Stellar winds, even in Wolf-Rayet stars are not able to carry oxygen away but the effect of mass loss is to increase the amount of lost helium and therefore to decrease the final amount of oxygen. Thus the net effect of a large mass loss is to increase the yields of $\mathrm{He}$ and decrease those of oxygen (see Maeder 1992). Oxygen is not affected by explosive nucleosynthesis whereas $\mathrm{Si}, \mathrm{S}$ and $\mathrm{Ca}$ are: these elements are also produced in a non-negligible way during the explosion of $\mathrm{SNe}$ Ia (the outcome of C-deflagration in a C-O white dwarf). On the other hand $\mathrm{O}$ and $\mathrm{Mg}$ are almost entirely produced in massive stars. The Fe-peak elements are mostly formed during explosive nucleosynthesis, mainly in type Ia SNe. In the explosive nucleosynthesis, the yields depend on the uncertain speed of the burning front and convection, and for type II SN explosions on the "mass-cut".

Here we recall the average $O$ yield from massive stars, obtained by integrating on the IMF, as derived from the most recent calculations (Woosley \& Weaver 1995; Thielemann, Nomoto \& Hashimoto 1996), as well as the yield of Fe from type Ia SNe (Thielemann et al. 1993). These yields are: $\left\langle y_{O}\right\rangle_{S N I I}=$ $1.77-0.59 M_{\odot},\left\langle y_{O}\right\rangle_{S N I a}=0.148 M_{\odot},\left\langle y_{F e}\right\rangle_{S N I I}=0.14-0.07 M_{\odot},\left\langle y_{F e}\right\rangle_{S N I a}=$ $0.744 M_{\odot}$. They clearly show that the bulk of $\mathrm{O}$ should originate from type II $\mathrm{SNe}$ whereas the bulk of Fe should originate from type Ia SNe.

\section{The Chemical Evolution Model}

We adopt the model of Chiappini et al. (1999) where the formation of the halo is disentangled from that of the disk. The halo and bulge form on a relatively short timescale (0.8-1.0 Gyr) after the first infall episode, whereas the disk accumulates much more slowly and "inside-out" during a second independent infall episode. The gas forming the disk is primordial. The adopted yields are from Woosley and Weaver (1995) and Thielemann et al. (1996) for massive stars, from van den Hoek and Groenewegen (1997) for low and intermediate mass stars and from Thielemann et al. (1993) for type Ia SNe. This model provides a very good fit to the G-dwarf metallicity distribution in the solar vicinity and requires a timescale for the formation of the disk in the solar neighbourhood of 7-8 Gyr. The model also provides a good fit to the solar abundances, the present time star formation rate, surface gas density, SN rates as well as abundance gradients and gas along the disk.

The data for oxygen from Gratton et al. $(1996,2000)$ show a slight increase of the $[\mathrm{O} / \mathrm{Fe}]$ ratio with decreasing $[\mathrm{Fe} / \mathrm{H}]$ at variance with what happens for other $\alpha$-elements which show a flatter plateau. This slight slope is well reproduced by theoretical models (Chiappini et al. 1999, 2000) due to the fact that the $\mathrm{O} / \mathrm{Fe}$ production ratio from massive stars, in the adopted yields, is an increasing function of the initial stellar mass and it varies more than for some of 
the other $\alpha$-elements. It is worth noting, in fact, that elements such as Si and $\mathrm{S}$ are not predicted to have a large overabundance relative to $\mathrm{Fe}$ in metal poor stars, due to the fact that they are also produced in a non-negligible way by type Ia SNe. The change in the slope, occurring at roughly $[\mathrm{Fe} / \mathrm{H}]=-1.0 \mathrm{dex}$, is due to the bulk of iron produced by type Ia SNe which becomes important after a timescale of the order of 1-1.5 Gyr. This change in slope corresponds also to the end of the halo phase and thus allows us to have an estimate for the duration of the halo-thick disk phase.

Although the predictions show an increase of the $[\mathrm{O} / \mathrm{Fe}]$ ratio, especially at very low metallicities, the predicted slope is still flatter than the observed one (Israelian et al. 1998) and the [O/Fe] is lower. This means that we cannot explain the rise of the $[\mathrm{O} / \mathrm{Fe}]$ in the framework of the standard nucleosynthesis. On the other hand, the standard nucleosynthesis can account for the oxygen abundance in the Solar System and for the $\mathrm{O}$ gradient along the disk (Chiappini et al. 2000).

\section{Conclusions}

We have described the evolution of the oxygen abundance relative to $\mathrm{Fe}$ in the Galaxy by means of a chemical evolution model which reproduces the majority of the observational constraints both in the local and whole disk. From the comparison between observations and model results we can draw the following conclusions:

-A successful model for the chemical evolution of the Galaxy (Chiappini et al. 1999,2000$)$ predicts that the $[\mathrm{O} / \mathrm{Fe}]$ ratio is roughly constant for stars with $[\mathrm{Fe} / \mathrm{H}]<-1.0$ but it increases for very low metallicities $([\mathrm{Fe} / \mathrm{H}]<-3.0)$ reaching values as high as $[\mathrm{O} / \mathrm{Fe}] \sim+0.9 \mathrm{dex}$. This value is too low if compared with recent data (Isrealian et al. 1998). The other $\alpha$-elements instead show a fairly constant plateau at low metallicities.

-The different behaviour of $[\mathrm{O} / \mathrm{Fe}]$, if real, cannot therefore be explained by the standard assumptions on nucleosynthesis and SN progenitors. If the same trend would be observed also for the other $\alpha$ elements we should revise the role of the different $\mathrm{SNe}$ in the Galactic enrichment. If only type Ia SNe would produce iron, in fact, the trend could be easily explained, although there are observations of SN remnants indicating that type II SNe do produce iron.

-Another possible explanation could be that there has been a generation of very massive pre-Galactic stars which have produced mainly oxygen (see also García López this meeting). On the other hand, the suggestion of Ramaty et al. (2000) that the increasing $[\mathrm{O} / \mathrm{Fe}]$ could be due to a delayed mixing of $\mathrm{Fe}$ would be valid only if the same effect were seen also in the other $\alpha$-elements.

\section{References}

Boesgaard, A.M., King, J.R., Deliyannis, C.P. \& Vogt, S.S., 1999, AJ, 117, 492 Chiappini, C., Matteucci, F., Beers, T.C., \& Nomoto, K. 1999, ApJ, 515, 226

Chiappini, C., Matteucci, F. \& Romano, D., 2000, ApJ, submitted 
Gratton, R.G., Carretta, E., Sneden, C. \& Matteucci, F., 1996, in Formation of the Galactic Halo... inside and out, ed. H. Morrison and A. Sarajedini, A.S.P. Conf. Ser. 92, p.307

Gratton, R.G., Carretta, E., Matteucci, F. \& Sneden, C. 2000, A\&A, 358, 671

Israelian, G., García López, R.J. \& Rebolo, R., 1998, ApJ, 507, 805

Maeder, A., 1992, A\&A, 264, 105

Ramaty, R., Scully, S.T., Lingenfelter, R.E. \& Kozlovsky, B., 2000, ApJ, 534, 747

Thielemann, F.K., Nomoto, K. \& Hashimoto, M. 1996, ApJ, 460, 408

Thielemann, F.K, Nomoto, K.\& Hashimoto, M., 1993, in Origin and Evolution of the Elements, ed by N. Prantzos et al., Cambridge Univ. Press, p.297

van den Hoek, L.B., Groenewegen, M.A.T. 1997, A\&AS, 123, 305

Woosley, S.E., Weaver, T.A. 1995, ApJS, 101, 181 